\title{
Pubmlst.org - the new home for the Borrelia MLSA database
}

\author{
Gabriele Margos ${ }^{1,2}$, Katrin Binder ${ }^{1}$, Eldina Dzaferovic ${ }^{1}$, Cecilia Hizo-Teufel ${ }^{1,2}$, Volker Fingerle ${ }^{1,2}$, \\ Andreas Sing ${ }^{1}$, Keith Jolly ${ }^{3}$
}

\author{
${ }^{1}$ Bavarian Health and Food Safety Authority, Oberschleissheim, Germany \\ ${ }^{2}$ German National Reference Centre for Borrelia, Oberschleissheim, Germany \\ ${ }^{3}$ Department of Zoology, University of Oxford, Oxford, UK
}

\begin{abstract}
For Borrelia, a multilocus sequence typing (MLST) scheme was developed based on sequence fragments of eight housekeeping loci (i.e. clpA, clpX, nifS, pepX, pyrG, recG, rplB, uvrA). The MLST/MLSA database accompanying the scheme has been moved to http://pubmlst.org/borrelia and now includes relapsing fever spirochetes. The new database is introduced here.
\end{abstract}

Multilocus sequence typing (MLST) and multilocus sequence analyses (MLSA) have been developed for epidemiological purposes, i.e. to aid understanding the relationship of bacterial pathogens at an intermediate time scale (Enright and Spratt, 1999; Urwin and Maiden, 2003). While MLST is used to resolve intraspecific relationships, MLSA has been recognized as suitable tools to address taxonomic questions, i.e. it is used at the genus level (Bishop et al., 2009). MLST and MLSA are based on the sequence information of internal fragments of about $500 \mathrm{bp}$ of housekeeping genes; often seven loci are chosen (Urwin and Maiden, 2003). The use of sequence information renders it unambiguous and portable. Even today traditional MLST and Sanger sequencing remains a fast and economical alternative to whole genome sequencing, in particular for bacteria that are difficult to culture (Jungnick et al., 2015; Mukhacheva and Kovalev, 2013).

In 2008, a Multilocus sequence typing (MLST) scheme was published for Borrelia burgdorferi sensu stricto that was based on eight housekeeping loci located on the main linear chromosome (Hoen et al., 2009; Margos et al., 2008). Because of the way the primers were designed, the scheme was extendable to other species of the Borrelia burgdorferi sensu lato species complex and used to study the populations within Borrelia genospecies and for taxonomic purposes (Hanincova et al., 2013; Jungnick et al., 2015; Margos et al., 2014; Margos et al., 2012; Margos et al., 2013; Mechai et al., 
2014; Mukhacheva and Kovalev, 2013; Ogden et al., 2011; Takano et al., 2011; Vitorino et al., 2008; Vollmer et al., 2013).

For all individual MLST and MLSA schemes specific databases maintain sequences, isolate specific information and tools for analyses (Aanensen and Spratt, 2005; Jolley et al., 2004; Perez-Losada et al., 2013). These databases are specifically designed to host MLST/MLSA data and serve to make these data available to the scientific community (Jolley, 2009). Since its development and introduction the Borrelia MLSA database was hosted and maintained at Imperial College London under http://borrelia.mlst.net. In February 2015 the database was moved to the Pubmlst.org website (http://pubmlst.org/databases) and extended to be used also for relapsing fever spirochetes. Here some features of the new interface are shown and described.

The new face of the Borrelia MLSA database is shown in Figure 1A. The website is divided into two parts: one that holds allele profile and sequence definitions and one that holds isolate data. Within each of the subdivisions, it is possible to browse, search and download data (Figure 1B). The database also offers the option to break down data according to their country of origin, species, year of collection and others (Figure 1C).

The move of the MLST/MLSA database for Borrelia was accompanied with an extension of the database to relapsing fever spirochetes. Primer for relapsing fever spirochetes were designed to conserved regions of the eight housekeeping loci, i.e. clpA, clpX, nifS, pepX, pyrG, recG, rplB, uvrA, using multiple alignments of $B$. duttonii strain Ly (GenBank accession no. NC_011229.1), B. hermsii strain DAH (GenBank accession no. CP000048.1), and B. turicatae strain 91E135 (GenBank accession no. CP000049.1). For primer design the program OligoExplorer v. 1.5 (http://www.genelink.com) was used. Primers were designed in such a way that the same fragments used for the Lyme borreliosis group of spirochetes could be sequenced and used in data analyses. The recently published detailed protocol for PCR conditions (Wang et al., 2014) is still valid and can also be found in the database.

Relapsing fever spirochetes are more diverse than LB spirochetes and therefore, degenerate primers had to be designed for the eight genes to allow amplification of the same region that was used for analyses of LB spirochetes. These primers were tested on a number of relapsing fevers spirochetes. DNA samples including B. recurrentis, B. duttonii, B. miyamotoi, and B. persica. Table 1 shows the primer sequences and results of successful amplification.

For relapsing fever species, the nifS primers showed such a high degree of degeneracy that they did not work for PCR amplification. For B. miyamotoi, specific primers for nifS amplification were designed using nifS sequences extracted from two fully sequenced genomes available in GenBank ( $B$. miyamotoi strain LB2001, GenBank accession no. NZ_CP006647.1/CP006647.2 and strain FR64, GenBank accession no NZ_CP004217.1/CP004217.1). In addition, the other degenerate primers showed little utility for sequencing PCR amplicons; therefore, specific sequencing primers were designed. For this, B. miyamotoi products amplified using degenerate primer were cloned into a pGEM-Vector (Promega, Mannheim, Germany) and sequenced using vector specific primers (SP6, T7). These sequences were used in multiple alignments in combination with $B$. miyamotoi sequences from GenBank to design sequencing primers that were specific for B. miyamotoi (Table 2). Multiple alignments were conducted in MEGA5 (Kumar et al., 2004).

Since $B$. miyamotoi occurs sympatrically with $B$. burgdorferi s.l., efforts were made to design primers that would not cross-react with $B$. burgdorferi s.l.. This was, however, not possible for all genes and 
primers that cross-react to $B$. burgdorferi s.l. are designated with * in Table 2. The application of MLSA to B. miyamotoi strains from Asia, USA and Europe showed that (i) loci can be amplified directly from ticks as the material from Germany constituted tick DNA extracts and (ii) the two $B$. miyamotoi strains from USA (LB2001 and M1029) did not differ in these loci supporting previous claims that local B. miyamotoi may be genetically very homogeneous (Bunikis et al., 2004; Richter et al., 2003).

For other relapsing fever spirochetes, only seven genes were amplified ( $c / p A, c l p X, p e p X, p y r G$, recG, $r p / B$ and $u v r A)$. The Pubmlst.org database, however, provides the option to submit incomplete allelic profiles. These incomplete profiles can be saved and connected to isolate data. Hence, for relapsing fever spirochetes, data for seven loci can be used to build phylogenetic trees and for other statistical analyses (e.g. genetic distance analyses). We suggest that the MLSA scheme described here will be a useful tool for population analyses of relapsing fever spirochetes which may be difficult to achieve using single locus approaches (Elbir et al., 2013). It may also be suitable for taxonomy in relapsing fever spirochetes.

\section{Acknowledgements}

The authors gratefully acknowledge the provision of B. miyamotoi strains by Hans Dautel, Insect Services, Berlin, Germany and Durland Fish, Yale University. The authors further acknowledge the excellent technical assistance of S. Stockmeier, German NRZ Borrelia. This work was partially funded by the RKI, Berlin, Germany via NRZ funding. VF, AS and GM are members of ESGBOR.

\section{References}

Aanensen, D.M. and Spratt, B.G. (2005) The multilocus sequence typing network: mlst.net. Nucleic Acids Res 33(Web Server issue): W728-733.

Bishop, C.J., Aanensen, D.M., Jordan, G.E., Kilian, M., Hanage, W.P. and Spratt, B.G. (2009) Assigning strains to bacterial species via the internet. BMC Biol 7(3).

Bunikis, J., Tsao, J., Garpmo, U., Berglund, J., Fish, D. and Barbour, A.G. (2004) Typing of Borrelia relapsing fever group strains. Emerg Infect Dis 10(9): 1661-1664.

Elbir, H., Raoult, D. and Drancourt, M. (2013) Relapsing fever borreliae in Africa. Am J Trop Med Hyg 89(2): 288-292.

Enright, M.C. and Spratt, B.G. (1999) Multilocus sequence typing. Trends Microbiol 7(12): 482-487. 
Hanincova, K., Mukherjee, P., Ogden, N.H., Margos, G., Wormser, G.P., Reed, K.D. et al. (2013) Multilocus sequence typing of Borrelia burgdorferi suggests existence of lineages with differential pathogenic properties in humans. PLoS One 8(9): e73066.

Hoen, A.G., Margos, G., Bent, S.J., Kurtenbach, K. and Fish, D. (2009) Phylogeography of Borrelia burgdorferi in the eastern United States reflects multiple independent Lyme disease emergence events. Proc Natl Acad Sci U S A 106: 15013-15018.

Jolley, K.A. (2009) Internet-based sequence-typing databases for bacterial molecular epidemiology. Methods Mol Biol 551: 305-312.

Jolley, K.A., Chan, M.S. and Maiden, M.C. (2004) mlstdbNet - distributed multi-locus sequence typing (MLST) databases. BMC Bioinformatics 5: 86.

Jungnick, S., Margos, G., Rieger, M., Dzaferovic, E., Bent, S.J., Overzier, E. et al. (2015) Borrelia burgdorferi sensu stricto and Borrelia afzelii: Population structure and Differential pathogenicity. International Journal of Medical Microbiology in press.

Kumar, S., Tamura, K. and Nei, M. (2004) Integrated software for Molecular Evolutionary Genetics Analysis and sequence alignment. Briefings in Bioinformatics 5: 150-163.

Margos, G., Gatewood, A.G., Aanensen, D.M., Hanincova, K., Terekhova, D., Vollmer, S.A. et al. (2008) MLST of housekeeping genes captures geographic population structure and suggests a European origin of Borrelia burgdorferi. Proc Natl Acad Sci U S A 105(25): 8730-8735.

Margos, G., Piesman, J., Lane, R.S., Ogden, N.H., Sing, A., Straubinger, R.K. and Fingerle, V. (2014) Borrelia kurtenbachii sp. nov., a widely distributed member of the Borrelia burgdorferi sensu lato species complex in North America. Int J Syst Evol Microbiol 64(Pt 1): 128-130.

Margos, G., Tsao, J.I., Castillo-Ramirez, S., Girard, Y.A., Hamer, S.A., Hoen, A.G. et al. (2012) Two boundaries separate Borrelia burgdorferi populations in North America. Appl Environ Microbiol 78(17): 6059-6067.

Margos, G., Wilske, B., Sing, A., Hizo-Teufel, C., Cao, W.C., Chu, C. et al. (2013) Borrelia bavariensis sp. nov. is widely distributed in Europe and Asia. Int J Syst Evol Microbiol 63(Pt 11): 4284-4288.

Mechai, S., Margos, G., Feil, E.J., Lindsay, L.R. and Ogden, N.H. (2014) Phylogeographic analysis reveals a complex population structure of Borrelia burgdorferi in southeastern and south central Canada. Appl Environ Microbiol.

Mukhacheva, T.A. and Kovalev, S.Y. (2013) Multilocus sequence analysis of Borrelia burgdorferi s.I. in Russia. Ticks Tick Borne Dis 4(4): 275-279. 
Ogden, N.H., Margos, G., Aanensen, D.M., Drebot, M.A., Feil, E.J., Hanincová, K. et al. (2011) Investigation of genotypes of Borrelia burgdorferi in Ixodes scapularis ticks collected in surveillance in Canada. Appl Environ Microbiol 77: 3244-3254.

Perez-Losada, M., Cabezas, P., Castro-Nallar, E. and Crandall, K.A. (2013) Pathogen typing in the genomics era: MLST and the future of molecular epidemiology. Infect Genet Evol 16: 38-53.

Richter, D., Schlee, D.B. and Matuschka, F.R. (2003) Relapsing fever-like spirochetes infecting European vector tick of Lyme disease agent. Emerg Infect Dis 9(6): 697-701.

Takano, A., Nakao, M., Masuzawa, T., Takada, N., Yano, Y., Ishiguro, F. et al. (2011) Multilocus Sequence Typing Implicates Rodents as the Main Reservoir Host of Human-Pathogenic Borrelia garinii in Japan. J Clin Microbiol 49(5): 2035-2039.

Urwin, R. and Maiden, M.C. (2003) Multi-locus sequence typing: a tool for global epidemiology. Trends Microbiol 11(10): 479-487.

Vitorino, L.R., Margos, G., Feil, E.J., Collares-Pereira, M., Ze-Ze, L. and Kurtenbach, K. (2008) Finescale Phylogeographic Structure of Borrelia lusitaniae Revealed by Multilocus Sequence Typing. PloS ONE 3(12): e4002.

Vollmer, S.A., Feil, E.J., Chu, C.Y., Raper, S.L., Cao, W.C., Kurtenbach, K. and Margos, G. (2013) Spatial spread and demographic expansion of Lyme borreliosis spirochaetes in Eurasia. Infect Genet Evol 14C: $147-155$.

Wang, G., Liveris, D., Mukherjee, P., Jungnick, S., Margos, G. and Schwartz, I. (2014) Molecular Typing of Borrelia burgdorferi. Current protocols in microbiology 34: 12C 15 11-12C 1531.

\section{Figure legends}

Figure 1A The new face of the Borrelia MLST database. Upon double click on "Borrelia spp " on the web site http://pubmlst.org/databases, the Borrelia MLST database opens. The database is divided into a "profile and sequence definitions" and "isolates". In profiles and sequence definitions, allele sequences, allelic profiles and sequence type information can be obtained as well as concatenated sequences of sequence types. The database "isolates" contains isolate specific information including strain name, year of isolation, geographic and biological origin, collector and submitter.

Figure 1B Browse, search and download functions are available inside the sequence or isolate specific databases. The figure shows the option to query the sequence database with an unknown gene sequence. 
Figure $1 \mathrm{C}$ In addition to search, browse and download sequences or isolate specific information, the database offers options to breakdown information contained in the database. The example provided shows a breakdown of isolates according to country of origin. 
Table 2 Borrelia miyamotoi MLSA primer

\section{PCR primers}

Gen direction Primer name Sequence/ Degeneracy, 5' to 3

clpA forward BmclpAF1268 TTGATCTCTTAGATGATCTTGG

reverse BmclpA2051R CAAACATAAACCTTTTCAGCCTTTAATA

clpX forward clpX104F CTGTTGCYATTTGTTTTGAATGYTC

reverse clpX1277R TAAAGTTCTTTTGCCCAAGG

nifS forward BmnifF31 GAAAAAGTAAACTCCCTCAGAAAGG

reverse BmnifS892R CAATGATGCCTGCAATATTTGGTG-3$$
\text { reverse }
$$

pepX forward

pep361F 5'-AGAGAYTTAAGYTTAKCAGG-3'

reverse pep1202R

5'-GTTTCTCTTAAAGAYTGCATTCC-3'

pyrG

forward pyrF415 5'-CTTYTAGTWATTGARATTGGTGGT

reverse pyr1261R 5'-CAGCATCAAYTATRCCACAAAC-3

recG forward rec908F* 5'-CTAGYATTCCTYTAATTGAGGC-3

reverse rec1779R* ${ }^{*}$-TTCRGTTAAAGGTTCCTTATAAAG-3

rplB forward rpl18F*

reverse rplR761* 5'-GGCTGNCCCCAAGGWGAT

uvrA forward uvr1170F* 5'-GAGGCGTTATCTTKCAACTG-3

reverse uvr2181R* ${ }^{*}$-AGACTCTGGAAGCTTWGC-3

forward uvrF1435 GCTKAAATTTTTRATTGATGTTGGA

reverse uvrR2306 5'-CARGGAACAAAAACATCRGGC

${ }^{*}$ cross reactivity with $B$. burgdorferi

\section{Sequencing primer}

direction Primer name

forward BmclpAF1268

reverse BmclpA2051R

forward BmclpF268

reverse $\quad B m c l p X 1155 R$

forward BmnifF31

reverse Bmnif892R

forward BmpepF358

reverse Bmpep1187R

forward BmpyrF417

reverse Bmpyr1252R

forward BmrecF909

reverse Bmrec1671R

forward

BmrplF23

Bmrpl759R

reverse

forward

reverse

BmuvrF1437
5' to 3 '

TTGATCTCTTAGATGATCTTGG

CAAACATAAACCTTTTCAGCCTTTAATA

TTATCTGTTGCTGTTTATAATC

TTCAAACATAACATCTTTAAGTAATTCTTC

GAAAAAGTAAACTCCCTCAGAAAGG

CAATGATGCCTGCAATATTTGGTG

GATAGAGACTTAAATTTAGCAGGAGTTG

TGCATTCCCCACATTGGAGTTC

TTTAGTAATTGAGATTGGTGGTC

TATTCCACAAACATTACGAGC

TAGCATTCCTTTAGTTGAGGC

CTCAGCATGCTCAACTACC

GACTTATAGGCCAAAAACTTC

GATACAGGATGACGACCACC

5'-TTAAATTTTTAAATTGATGTTGGACT

Bmuvr2147R 
Table 1 MLSA Primer relapsing fever spirochetes

\section{approximate}

\begin{tabular}{|c|c|c|c|c|}
\hline gene & $5^{\prime}$ position & primer name & Sequence/degeneracy $5^{\prime}$ to $3^{\prime}$ & $\begin{array}{l}\text { Remarks } \\
\text { worked only for }\end{array}$ \\
\hline \multirow[t]{2}{*}{ clpAF } & 1258 & clpAF1258 & GATAAAGCTTTTGAYYTATTAGATGG & B. recurrentis \\
\hline & 1262 & clpAF1262 & AAGCTTTTGAYYTATTAGATGGTC & \\
\hline \multirow[t]{2}{*}{ clpAR } & 2098 & clpAR2098 & \multicolumn{2}{|l|}{ CAAAAAAAASATCHARRTTTTCATTTTTTAG } \\
\hline & 2276 & clpAR2276 & TCATATTTDATRGTDTCGTC & B. recurrentis \\
\hline \multirow[t]{3}{*}{ clpXF } & 104 & clpXF104 & CTGTTGCYATTTGTTTTGAATGYTC & $\begin{array}{l}\text { sometimes non-specific } \\
\text { bands }\end{array}$ \\
\hline & 109 & clpXF109 & GCYATTTGTTTTGAATGTTCTAAAATATG & \\
\hline & 268 & clpXF268 & TTRTCYGTTGCTGTTTATAATC & \\
\hline \multirow[t]{2}{*}{ clpXR } & 1183 & clpXR1183 & CTTTTTTAATTTGCTTASTWGAAGG & \\
\hline & 1277 & clpXR1277 & TAAAGTTCTTTTGCCCAAGG & \\
\hline
\end{tabular}

non-specific bands in combination with pepR 1187

\begin{tabular}{|c|c|c|c|}
\hline \multirow[t]{2}{*}{ pepXF } & 361 & pepF361 & AGAGAYTTAAGYTTAKCAGG \\
\hline & 364 & pepF364 & GAYTTAAGYTTAKCAGGARTTG \\
\hline \multirow[t]{3}{*}{ pepXR } & 1187 & pepR1187 & TGCATTCCCCACATTGG \\
\hline & 1202 & pepR1202 & GTTTCTCTTAAAGAYTGCATTCC \\
\hline & 1207 & pepR1207 & CYATAGTTTCTCTTAAAGAYTGC \\
\hline \multirow[t]{2}{*}{ pyrGF } & 372 & pyrF372 & TGGRAARTATTTAGGKAGAACTG \\
\hline & 379 & pyrF379 & TATTTAGGKAGAACTGTACAGC \\
\hline \multirow[t]{3}{*}{ pyrGR } & 1252 & pyrR1252 & TATRCCACAAACATTACGKGC \\
\hline & 1261 & pyrR1261 & CAGCATCAAYTATRCCACAAAC \\
\hline & 1375 & pyrR1375 & CAAGTCGCATTGTWGCAC \\
\hline \multirow[t]{2}{*}{ recGF } & 898 & recF898 & GСKTTTCTMTCTAGYATTCC \\
\hline & 908 & recF908 & CTAGYATTCCTYTAATTGARGC \\
\hline recGR & 1779 & recR1779 & TTCRGTTAAAGGTTCCTTATAAAG \\
\hline \multirow[t]{3}{*}{ rplBF } & 3 & rplF3 & GGAGAAAAATATGGGKATTAAGAC \\
\hline & 18 & rplF18 & ATTAAGACTTATARGCCAAAAAC \\
\hline & 24 & rplF24 & GACTTATARGCCAAAAACTTC \\
\hline \multirow[t]{3}{*}{ rplBR } & 759 & rplR759 & GATACAGGATGWCGACC \\
\hline & 765 & rplR765 & CCAAGGWGATACAGGATG \\
\hline & 769 & rplR769 & GRCCCCAAGGWGATAC \\
\hline
\end{tabular}




\begin{tabular}{llll} 
uvrAF & 1170 & uvrF1170 & GAGGCGTTATCTTWCAAC \\
& 1173 & uvrF1173 & GCGTTATCTTWCAACTGAATC \\
\multirow{4}{*}{ uvrAR } & 2147 & uvrR2147 & TCDGTAAAAAATCCAACATAAGTTGC \\
& 2153 & uvrR2153 & CTAATCTCDGTAAAAAATCCAACATAAG \\
& 2178 & uvrR2178 & TCTAGACTCTGGAAGCTT
\end{tabular}

sometimes non-specific bands with uvrR2147 